\title{
Adolescent-onset psychosis: A 2-year retrospective study of adolescents admitted to a general psychiatric unit
}

S Paruk, MB ChB, FCPsych (SA), Certificate in Child Psychiatry

S Ramlall, MB ChB, FCPsych (SA)

J K Burns, MB ChB, FCPsych (SA), MSc (Edinburgh)

Department of Psychiatry, Nelson R Mandela School of Medicine, University of KwaZulu-Natal, Durban

Background. KwaZulu-Natal had no dedicated inpatient adolescent psychiatric service during the study period, and adolescents were admitted to general psychiatric wards.

Aim of study. This is a descriptive review of adolescents with psychotic symptoms admitted to a psychiatric hospital. It aims to describe their demographic profile, associated risk factors and clinical profile, and management strategies utilised.

Method. The files of all adolescent patients, aged $12-18$ years, admitted to a psychiatric hospital with psychotic symptoms from July 2005 to June 2007 were reviewed.

Results. Seventy adolescents with psychosis were admitted to adult psychiatric wards over the 2-year period. The age range was $13-18$ years. Of the patients $80.0 \%$ were male, $37.1 \%$ reported a positive family history of mental illness, $50.0 \%$ smoked nicotine and $61.4 \%$ reported cannabis use. The most common diagnoses were schizophrenia (30.0\%) and schizophreniform disorder (27.1\%). Sixty $(85.7 \%)$ of the patients had a trial on a firstgeneration antipsychotic and $10(15.5 \%)$ were initiated on a second-generation antipsychotic de novo. The average length of stay in hospital was 27.8 days. Of the patients, $40 \%$ defaulted follow-up after discharge.

Conclusions. Schizophrenia was the most common diagnosis. There were high rates of cannabis use. The adolescents were managed in general psychiatric wards. The majority of patients had a trial on first-generation antipsychotics. There was a high rate of defaulting the first outpatient appointment. There is a need to develop specialised inpatient adolescent psychiatric facilities and services, as well as to address comorbid substance use and non-adherence to treatment.
The Diagnostic and Statistical Manual of Mental Disorders, 4th edition, text revision (DSM-IV-TR) defines psychosis as having delusions or hallucinations, with the hallucinations occurring in the absence of insight into their pathological nature. A broader definition includes symptoms such as disorganised speech and grossly disorganised or catatonic behavior.' Semper and McClellan refer to the DSM definition of psychosis for children and also note that negative symptoms such as alogia, amotivation and anhedonia can be present. ${ }^{2}$ Cognitive and mood symptoms may also be present. The DSM-IV-TR applies the same diagnostic criteria for psychotic disorders in children and adolescents as for adults.

There are relatively few epidemiological studies on adolescentonset psychosis. It is generally held that the incidence of psychosis, and especially of schizophrenia, increases markedly during the teenage years, with a preponderance of male over female patients. ${ }^{3}$

Risk factors for early-onset psychosis (before age 18) relate to the interaction of environmental and biological factors in vulnerable individuals. Weiden and Buckley suggest that urban residence, social adversity and early substance use, specifically cannabis use, are environmental risk factors that may increase the risk of developing schizophrenia during adolescence and young adulthood. ${ }^{4} \mathrm{~A}$ positive family history, birth complications, advanced parental age, childhood developmental abnormalities and early infections are some of the biological factors that have been reported. ${ }^{5}$ Dalman et al. reviewed exposure to infections in early life as a risk factor for the development of psychosis. ${ }^{6}$

There is often diagnostic uncertainty in the first episode of adolescent-onset psychosis. Key diagnostic issues relate to distinguishing schizophrenia from bipolar disorder in psychotic adolescents. There is also the concern that criteria for diagnosis of schizophrenia in adults do not apply directly to children and adolescents. The differential diagnosis may include schizophrenia, bipolar disorder, psychotic disorder due to a general medical condition, or a substance-induced psychotic disorder. Delusional disorder and brief psychotic disorder are less common. ${ }^{8}$ 
The evidence for antipsychotic use in adolescents is limited but increasing gradually. Treatment guidelines generally recommend a combination of antipsychotics and psychosocial interventions. The advent of atypical antipsychotics agents has enhanced the potential for effective treatment. ${ }^{9}$

This study describes the local demographic profile, clinical features, associated risk factors and management strategies utilised among adolescents presenting with psychosis and admitted to a psychiatric hospital. The following variables were also assessed for statistical associations: (i) positive family history and age of presentation; (ii) substance use and age of presentation; and (iii) length of hospital stay and substance use. The correlation between length of hospital stay and duration of symptoms before presentation was also assessed.

\section{Methodology}

The study was a descriptive, naturalistic retrospective chart review of adolescents with psychotic symptoms who were admitted to a psychiatric institution in Durban, KwaZulu-Natal. At the time of the study, the province of KwaZulu-Natal had no inpatient adolescent facility, and adolescent patients were admitted to adult psychiatric wards. All patients meeting the inclusion criteria were identified through the hospital admission records. Participants included in the study were 12 - 18-year-old adolescents who were hospitalised with psychotic symptoms during the period 1 July 2005 - 30 June 2007. All had been assessed by a psychiatrist during their admission.

A structured data sheet was used to collate the data, all files having been reviewed by the principal investigator (S P).

Demographic data collected from the medical records included age, gender, religion, ethnicity, language and educational level. Information on social background was also obtained. Data on risk factors such as family history, medical history, HIV status (if known), substance use, past psychiatric history, mental retardation, forensic history, and a history of abuse, neglect or any other documented psychosocial stress were recorded. Clinical data included a multi-axial diagnosis, duration of symptoms, age of onset of first-episode psychosis, investigations recorded, legal status of admission in terms of the Mental Health Care Act, length of hospitalisation, medications prescribed and adherence to first follow-up visit.

Data were analysed using SPSS version 15.0 (SPSS Inc; Chicago, III., USA). Pearson's chi-square test was used to test associations between variables with categorical outcomes, while analysis of variance (ANOVA) was used for quantitative normally distributed outcomes. A p-value of $<0.05$ was considered statistically significant.

Ethics approval was obtained from the Biomedical Research Ethics Committee of the University of KwaZulu-Natal, and permission to conduct the study was given by the provincial Department of Health and the hospital.

\section{Results}

A total of 70 adolescents were admitted to adult psychiatric wards over the 2-year period.

\section{Demographic profile}

The age range was $13-18$ years, with a mean of 16.79 /standard deviation (SD) 1.273) years. Of the patients, $80.0 \%$ (56) were male; $84.3 \%$ (59) were black, 10\% Indian and 5.7\% coloured; $72.1 \%$ (49) were of the Christian faith; $72.6 \%$ (51) spoke isiZulu and $18.6 \%(13)$ spoke English as their first language; and $85.7 \%$ (60) were living at home with family, 10.0\% (7) in institutions and 1 with a foster family, while 2 had no fixed abode at the time of admission. Of the patients, $27.9 \%(17 / 61)$ were born in the South African winter months of June, July and August. The dates of birth for 9 patients were not available.

\section{Associated risk factors}

A positive family history of mental illness was reported by $37.1 \%$ (26) of the patients; $50.0 \%$ (35) of the adolescents smoked nicotine, $61.4 \%$ (43) admitted to cannabis use and $41.4 \%$ (29) reported alcohol use, in the past or at the time of admission; and $47.1 \%$ (33) reported adverse psychosocial stressors currently or in the past. The most common stressor described was the loss of a significant caregiver.

Six $(8.6 \%)$ of the sample were mentally retarded and $22(31.4 \%)$ had a positive forensic history prior to admission, with charges varying from theft to assault to rape.

\section{Clinical profile}

Forty-five (64.3\%) of the adolescents were admitted with a first episode of psychosis while 25 (35.7\%) had a past history of psychosis. The data on the sub-cohort of adolescents presenting with a first episode of psychosis will be reported on separately. The most common diagnoses in the present study were schizophrenia (30\%) and schizophreniform disorder (27.1\%). Table I illustrates 
the frequency of the different Axis I diagnoses in the adolescents presenting with psychosis.

A co-morbid Axis I diagnosis was made in 28.6\% (20) patients, the most common being a substance-related disorder; $64.3 \%$ (45) had a co-morbid medical diagnosis on Axis III, the most common being epilepsy (8.5\%).

The results of basic haematological tests performed were generally normal, as summarised in Table $\|$.

Thyroid function tests were performed in 32 patients and were abnormal in 3 (4.3\% of the total), and of 32 results of serological tests for syphilis reported, 1 was abnormal.

Of the 70 patients 38 had computed tomography (CT) scans of the brain, and 7 of these scans revealed abnormalities. The abnormalities reported are summarised in Table II.

HIV status was not known in $95.7 \%$ of patients. HIV results were only available for 3 of the 70 files audited, with 1 patient being HIV positive.

\section{Management}

All the patients were prescribed antipsychotics; 60 (85.7\%) patients had a trial on a first-generation antipsychotic, and only 10 (14.5\%) were initiated on a second-generation antipsychotic de novo. A benzodiazepine was required for sedation in 41 cases $(58.6 \%)$, and 29 patients $(41.4 \%)$ received anticholinergic medication at some stage during admission. One patient received electroconvulsive treatment.

While $85.7 \%$ of the patients were initiated on typical antipsychotics, $27(45.8 \%)$ of the 59 patients who were discharged from the unit were discharged on atypical antipsychotics. Eleven patients were

\begin{tabular}{lc} 
Table II. Haematological test results \\
\hline Haematological test & \% normal \\
\hline Full blood count & 97.1 \\
Urea and electrolytes & 95.7 \\
Liver function tests & 97.1 \\
Random glucose & $81.4^{*}$ \\
*11 7\% of patients had no record of blood glucose testing. \\
\hline
\end{tabular}

\section{Table III. CT scan abnormalities}

\begin{tabular}{lc} 
Abnormality & Frequency $(N)$ \\
\hline Arachnoid cyst & 2 \\
Cerebral atrophy (generalised) & 1 \\
Left temporal lobe infarction & 1 \\
Calcified granuloma & 1 \\
Multiple calcified densities bilaterally & 1 \\
Temporal lobe atrophy & 1 \\
\hline
\end{tabular}

transferred to another psychiatric unit and their discharge data were not available.

The average length of stay in hospital was 27.8 days (SD 23.8), and $94.3 \%(66)$ of the patients were admitted as involuntary mental health care users. Of those patients who were to be followed up at the unit, $60 \%$ attended the first visit after discharge; $31.3 \%$ of patients were prescribed typical antipsychotics and $44.4 \%$ of patients on atypical antipsychotics defaulted treatment, but this difference was not clinically significant $(p=0.492)$.

\section{Associations}

There were no statistically significant associations between any of the following variables: positive family psychiatric history and age of presentation, substance use history and age of presentation,

\section{Table I. Diagnoses in adolescents with psychosis}

\begin{tabular}{|c|c|c|}
\hline & Frequency $(N)$ & $\%$ \\
\hline Brief psychotic disorder & 4.0 & 5.7 \\
\hline Schizophreniform disorder & 19.0 & 27.1 \\
\hline Schizophrenia & 21.0 & 30.0 \\
\hline Psychotic disorder due to general medical condition & 7.0 & 10.0 \\
\hline Substance-induced psychotic disorder & 8.0 & 11.4 \\
\hline Schizo-affective disorder & 3.0 & 4.3 \\
\hline Major depressive disorder with psychotic features & 2.0 & 2.9 \\
\hline Bipolar disorder - manic with psychotic features & 5.0 & 7.1 \\
\hline Psychotic disorder not otherwise specified & 1.0 & 1.4 \\
\hline Total & 70.0 & 100.0 \\
\hline
\end{tabular}


duration of hospitalisation and substance use. The adolescents with positive substance use histories tended to have a slightly longer duration of hospitalisation, but this was not statistically significant.

There was no statistically significant correlation between length of hospital stay and duration of symptoms before admission.

\section{Discussion}

While psychotic disorders are considered rare in adolescents, they may severely affect development and long-term functioning. Early identification and intervention are critical to improving outcome. This chart review suggests that a considerable number of adolescents presenting with psychosis required lengthy periods of inpatient care as involuntary mental health care users in adult psychiatric wards.

\section{Demographic profile}

In this study, there was a preponderance of male adolescents with psychosis. This was also borne out in the Canadian Child and Adolescent First Episode Psychosis study (CAFEPS). ${ }^{10}$ The majority of adolescents in our study were black Africans. In a recent British study, adolescents from the black ethnic group were over-represented among those admitted with a diagnosis of psychotic disorder. " Ethnic and cultural factors may influence the clinical presentation, response to treatment and prognosis of schizophrenia. ${ }^{12}$ Emsley et al. suggest that while the literature on ethno-psychopharmacology studies has reported racial disparities in dose, route of delivery and class of antipsychotic prescribed Iwith African Americans more likely to receive higher doses and depot antipsychotics), this was not borne out in their own research locally. ${ }^{12}$

The ethnic demographics in the present study may reflect the population served. The local demographic profile should be considered in the planning of future services and prevention programmes so that they are appropriate and relevant. This has several implications: $80 \%$ of the adolescents spoke isiZulu as their first language, yet most mental health care professionals speak English. Patient and family interviews and therapy such as psycho-education should be provided in the patient's first language to facilitate communication. Further, as there are strong cultural influences on value systems, family hierarchies of authority, attribution of causality and hence acceptability of treatment interventions, recognising and addressing existing linguistic barriers and cultural biases are critical in providing appropriate and effective care in multi-cultural settings.

\section{Risk factors}

Risk factors for the development of psychosis are multi-factorial. The interplay between biological factors such as genetic vulnerability (suggested by a positive family history of mental illness), substance use, epilepsy and psychosocial stressors is evident in our study findings. Specifically, we observed high rates of substance use, psychosocial stressors, mental retardation and positive family history of mental illness.

A positive family history of mental illness supports the theory of a genetic vulnerability for the development of psychosis. Although a number of genes have been associated with schizophrenia, the exact genetic mechanisms of schizophrenia are still unclear. ${ }^{13}$ Van Os proposes that while genetic factors contribute to the schizophrenia phenotype, the higher concordance rate for schizophrenia in monozygotic twins compared with dizygotic twins may in part be due to their sharing the same early environmental risk factors. ${ }^{14}$

In a recent study on childhood IQ and adult mental disorders, low $I Q$ was an antecedent of many psychiatric disorders including schizophrenia spectrum disorders. Low $I Q$ is also considered predictive of persistence and co-morbidity. ${ }^{15}$

While the evidence is conflicting, it has been suggested that infections in early life lespecially fetal and early childhood infections) play a role in the causation of schizophrenia. Births in winter are postulated as a risk factor, as neurotropic infections may contribute to schizophrenia in some cases. ${ }^{6}$ In a Third-World setting, many children are exposed to a variety of infections during their early developmental years, and the possible role of these infections in the development of schizophrenia in adolescents requires further study. A larger sample would be required to clarify the role of infections in our local population.

Our results suggest a high prevalence of self-reported substance use (including nicotine, cannabis and alcohol) and are consistent with the literature. Weiser et al. reported a $12.4 \%$ prevalence of self-reported drug abuse in adolescent males with behavioural disturbances who were later hospitalised for schizophrenia, as opposed to a $5.9 \%$ prevalence in adolescent males who were not hospitalised later. ${ }^{16}$ In their study, this association was specific for schizophrenia and all non-affective psychotic disorders, and was independent of cognitive functioning, social functioning and the presence of a non-psychotic disorder in the Draft Board assessment by the Israeli military. ${ }^{16}$ Rates of substance use are high in psychotic adolescents, and this may be an attempt to selfmedicate early symptoms. Alternatively, cannabis may precipitate the psychotic illness. 
Henquet et al. suggest that mechanisms of gene-environment interaction underlie the association between cannabis and psychosis. In this respect, multiple variations within multiple genes (rather than a single genetic polymorphism) together with other environmental factors may interact to increase the risk of psychosis. $^{17}$

The risk of developing psychosis is thought to increase with longer duration of exposure to cannabis, with adolescents at particular risk. The adolescent brain is vulnerable, as it is still developing. ${ }^{18}$ Substance use and especially misuse has significant implications for the management, course and prognosis of psychosis.

\section{Clinical profile}

The differential diagnosis in children and adolescents presenting with psychotic symptoms is broad and includes primary psychotic disorders such as schizophrenia and schizo-affective disorder, and psychotic mood disorders such as bipolar mood disorder and psychotic depression. ${ }^{2}$ In the present study, primary psychotic disorders were the most common diagnosis and only 7 patients were diagnosed with a mood disorder. The diagnostic spread in the present study is reflective of this spectrum, and diagnoses may evolve over time.

Co-morbid psychiatric and medical disorders tend to correlate with a poorer outcome. Patients with severe mental illness have higher than expected prevalence rates of co-morbid general medical conditions, particularly metabolic and cardiovascular disease. They are also at increased risk of contracting HIV infections. Conversely, these and other medical disorders also increase the risk of developing mental disorders. ${ }^{19}$ A clinically significant level of medical co-morbidity and psychotic disorder due to general medical disorder was detected in our study, reinforcing the need for comprehensive screening

The estimated HIV seroprevalence ranges from $5 \%$ to $20 \%$ in patients with psychosis. ${ }^{20}$ A study by Mashaphu and Mkize at a psychiatric institution in KwaZulu-Natal found a 23.8\% HIV seroprevalence rate in adults with first-episode psychosis. ${ }^{20}$ In the current study, HIV results were available for only 3 patients. The lack of HIV testing may be due to patient refusal or to deferment of testing until the patient is competent to provide consent. The low level of HIV testing is of concern in a province where the prevalence of HIV infection is very high and the prevalence among adolescents with psychosis is not known. Early recognition of HIV infection in a high-risk and very vulnerable clinical population is necessary if the neuropsychiatric sequelae are to be treated more appropriately and effectively through the use of antiretroviral agents and the overall prognosis is to be improved.
Psychotic disorder due to a general medical condition may have been under-diagnosed in the current study because of deficiencies in the investigation of the adolescent patients, as HIV testing was generally not done, many patients (38) did not have thyroid tests done or results recorded, and only 38 of the 70 patients had CT brain scans.

Investigations in early adolescent psychosis should serve to exclude general medical conditions. In the absence of abnormal findings on physical examination, CT scanning or magnetic resonance imaging are unlikely to detect any underlying neurological condition. ${ }^{3}$

Nevertheless, structural neuro-imaging studies have shown subtle abnormalities in psychotic adolescents. Moreno et al. reported that male adolescents with a first psychotic episode showed significantly larger volumes of overall cerebrospinal fluid (CSF) and left frontal and right parietal sulci CSF. Male patients also showed significantly lower volumes of grey matter in the right and left frontal lobes. No significant volumetric differences were found in females. ${ }^{21}$ The Moreno study concluded that larger CSF and lower grey matter volumes in the frontal lobes may be a nonspecific vulnerability marker for psychosis in male adolescents. ${ }^{21}$ In our study, 7 of the scans showed abnormalities. Although some abnormalities were not sufficiently clinically significant to warrant any further intervention, neuro-imaging remains an important investigation to detect subtle intracranial abnormalities that may otherwise be missed.

There is evidence to suggest that patients with severe mental illness are at increased risk of developing abnormal glucose homeostasis independent of treatment with medication. ${ }^{19}$ Studies have mainly focused on atypical antipsychotics, and there is relatively little information on first-generation antipsychotics. ${ }^{19}$ In the present, study the patients tested had normal random blood glucose levels. Clinicians need to monitor closely for the development of metabolic syndrome. Protocols for screening and regular monitoring of weight, abdominal circumference, glucose, cholesterol and triglycerides in these young patients need to be implemented.

\section{Management}

Atypical antipsychotics are commonly considered the treatment of choice in children and adolescents based on assumptions of efficacy and tolerabilty. ${ }^{22}$ In the state sector in South Africa, and specifically in KwaZulu-Natal, conventional antipsychotics are still widely used as first-line treatment. This was borne out in the current study. Furthermore $41.4 \%$ of the patients required anticholinergic medication, suggesting that they had developed extra-pyramidal 
side-effects. There is a need for treatment guidelines that consider local factors (such as availability, accessibility and affordability of treatment) for the management of adolescents with psychosis.

Treatment adherence is another critical challenge in preventing relapse and readmission. The Clinical Antipsychotic Trials of Intervention Effectiveness (CATIE) trial, which examined the efficacy of four second-generation antipsychotics and one first-generation antipsychotic, showed that $74 \%$ of subjects discontinued their treatment before the end of the trial (18 months). There was no significant difference between the drugs in time to discontinuation due to intolerable side-effects. ${ }^{23}$ This was also borne out in our study in that there was no significant difference in the outpatient follow-up rate between patients on first- or second-generation antipsychotics. The high rate of discontinuation of treatment in the present study reiterates the need for psycho-education of patients and families and comprehensive case management that also addresses psychosocial issues. Unfortunately, psychosocial interventions require extensive resources, and while initial results have been promising, sustained efficacy has not been confirmed in later studies in psychotic patients. ${ }^{24}$ Owing to scarce resources, psychosocial interventions for psychotic patients are often very limited or even neglected in our setting.

All the patients in our sample were admitted to adult psychiatric wards because of a lack of dedicated adolescent facilities. The hospital has since opened an adolescent inpatient unit. Boeing et al. confirmed the widely held clinical impression that there is a gap in adolescent inpatient care provision, with $80 \%$ of first admissions being admitted into adult wards. ${ }^{25}$ Adolescents in adult wards pose numerous risks, ethical concerns and therapeutic limitations, and this shortcoming in mental health facilities needs to be addressed.

\section{Limitations}

The study was a retrospective chart review and relied on medical records for data collection. Omissions or inaccuracies in the records would compromise the results of the study. Nonetheless, this study provides a valuable 'snapshot' of psychosis in adolescents. The small sample size of 70 patients may account for our negative correlation results.

The site of the study is a tertiary-level psychiatric hospital, which presents a bias towards severity of symptoms or aggression, which are the main reasons for admission. However, the site does provide a fair representation of adolescents admitted with psychosis, as psychiatric services in the region are generally scarce and unevenly distributed.

\section{Conclusion}

Schizophrenia was the most common diagnosis, and there were high rates of cannabis use among the psychotic adolescents. Most of the patients were male and isiZulu-speaking. The adolescents were all managed in adult psychiatric wards, and the majority of patients were initiated on first-generation antipsychotics. A deficiency in HIV screening was noted, and a significant number of patients defaulted treatment. The study therefore suggests that adolescents presenting with psychosis are a vulnerable clinical group that has significant psychopathology and requires intensive and age-appropriate specialist management.

Comprehensive evaluation, which includes identifying and addressing risk factors and investigating the patient to exclude general medical conditions, should be considered part of routine care. There needs to be special focus on enhancing treatment adherence and addressing co-morbid substance use in order to improve long-term outcome. Atypical antipsychotics should be considered as first-line treatment in adolescents with psychosis where possible.

Child and adolescent psychiatric services need to be prioritised. There is a need to promote mental health programmes for adolescents and specifically to provide in- and outpatient services for psychotic adolescents. The local demographic factors, associated risk factors and clinical profile and the availability of different treatment options should be considered in planning future services and local treatment guidelines.

This study has provided a descriptive review, and further research is required on the epidemiology, risk factors, clinical features, management and prognostic factors of psychosis in children and adolescents. Better understanding of the many complexities in adolescents with psychosis will translate into enhanced treatment outcomes.

\section{References}

1. American Psychiatric Association. Diagnostic and Statistical Manual of Mental Disorders. 4th ed., text revision. Washington, DC: American Psychiatric Association, 2000

2. Semper TF, McClellan JM. The psychotic child. Child Adolesc Psychiatr Clin North Am 2003; 12: 679-691

3. Clark AF. Proposed treatment for adolescent psychosis: Schizophrenia and schizophrenia like psychoses. Advances in Psychiatric Treatment 200 1; 7: 16-23.

4. Weiden PJ and Buckley PF. Understanding and treating 'first-episode' schizophrenia Psychiatr Clin North Am 2007; 30(3): 48 1-506.

5. Messias EL, Chen CY, Eaton WW. Epidemiology of schizophrenia: review of findings and myths. Psychiatr Clin North Am 2007; 30(3): 323-338.

6. Dalman C, Alleback P, Gunnel D, et al. Infections in the CNS during childhood and the risk of subsequent psychotic illness: A cohort study of more than one million Swedish subjects. Am J Psychiatry 2008; 165(1): 59-65.

7. Schulz SC, Findling RL, Wise A, Friedman L, Kenny J. Child and adolescent schizophrenia. Psychiatr Clin North Am 1998; 21 (1): 43-56 
8. Reimherr JP, McClellan JM. Diagnostic challenges in children and adolescents with psychotic disorders. J Clin Psychiatry 2004; 65: suppl. 6, 5-1 1 .

9. American Academy of Child and Adolescent Psychiatry. Practice parameters for the assessment and treatment of children and adolescents with schizophrenia. J Am Acad Child Adolesc Psychiatry 2001; 40: suppl. 7, 4S-23S.

10. Castro-Fornieles J, Parellada M, Gonzalez-Pinto A, et al. The child and adolescent first episode psychosis study (CAFEPS): Design and baseline results. Schizophr Res 2007; 91: 226-237.

11. Tolmac J, Hodes M. Ethnic variation among adolescent psychiatric in-patients with psychotic disorders. BrJ Psychiatry 2004; 184: 428-431.

12. Emsley R, Oosthuizen $P$, Niehaus D, Koen L, Chiliza B. Changing the course of schizophrenia - predictors of treatment outcome revisited. South African Journal of Psychiatry 2007; 13(1): 4-9.

13. Sanders AR, Duan J, Levinson DF, et al. No significant association of 14 candidate genes with schizophrenia in a large European ancestry sample: implications for psychiatric genetics. Am J Psychiatry 2008; 165: 497-506.

14. Van Os J. Genotype-environment interaction and psychosis: from theory to genuine public health issue? Schizophrenia Monitor 1998; 8(4): $51-54$

15. Koenen KC, Moffit TE, Roberts AL, et al. Childhood IQ and adult mental disorders: A test of the cognitive reserve hypothesis. Am J Psychiatry 2008; 166(1): 50-57.

16. Weiser $M$, Reichenberg A, Rabinowitz J, et al. Self reported drug abuse in male adolescents with behavioural disturbances, and follow-up for future schizophrenia. Biol Psychiatry 2003; 54: 655-660
17. Henquet C, Di Forti M, Morrison P, Kuepper R, Murray RM. Gene-environment interplay between cannabis and psychosis. Schizophr Bull 2008; 34(6): 1111 1121.

18. Van Os J, Bak M, Hanssen M, Bijl RV, de Graaf R, Verdoux H. Cannabis use and psychosis: a longitudinal population based study. Am J Epidemiol 2002; 156(4): 319-327.

19. Oosthuizen P, Carey P and Emsley RA. Psychiatric disorders and general medical conditions: implications for the clinician. African Journal of Psychiatry 2008; 11 (1) $18-22$

20. Mashaphu S and Mkize DL. HIV seropositivity in patients with first episode psychosis. South African Journal of Psychiatry 2007; 13(3): 90-94.

21. Moreno $D$, Burdalo $M$, Reig $S$, et al. Structural neuroimaging in adolescents with first psychotic episode. J Am Acad Child Adolesc Psychiatry 2005; 44(1 1): 1151 1-1 157.

22. Sikich L, Frazier JA, McClellan J, et al. Double-blind comparison of first-and secondgeneration antipsychotics in early-onset schizophrenia and schizoaffective disorder: Findings from the treatment of early onset schizophrenia spectrum disorders (TEOSS) study. Am J Psychiatry 2008; 165: 1420-1430.

23. Lieberman JA, Stroup TS, McEvoy JP, et al. Effectiveness of antipsychotic drugs in patients with chronic schizophrenia. N Engl J Med 2005; 353(12): 1209-1223.

24. Byerly MJ, Nakonezny PA, Lescouflair E. Antipsychotic medication adherence in schizophrenia. Psychiatr Clin North Am 2007; 30: 437-450.

25. Boeing L, Murray V, Pelosi A, McCabe R, Blackwood D, Wrate R. Adolescent onse psychosis: prevalence, needs and service provision. BrJ Psychiatry 2007; 190: 18 26 